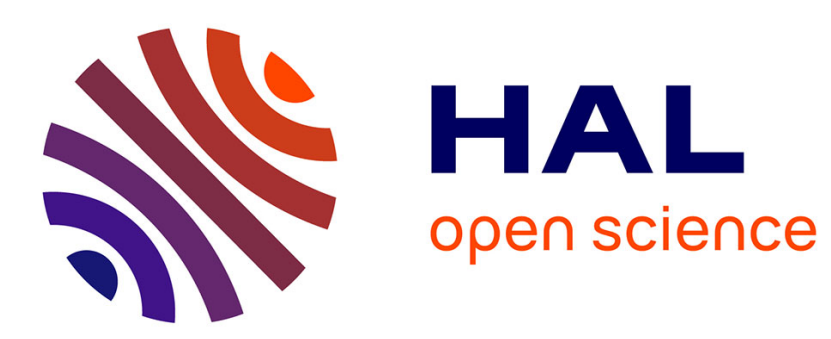

\title{
Mental simulation of future scenarios in transient global amnesia.
}

\author{
Aurelija Juskenaite, Peggy Quinette, Béatrice Desgranges, Vincent de La \\ Sayette, Fausto Viader, Francis Eustache
}

\section{- To cite this version:}

Aurelija Juskenaite, Peggy Quinette, Béatrice Desgranges, Vincent de La Sayette, Fausto Viader, et al. Mental simulation of future scenarios in transient global amnesia.. Neuropsychologia, 2014, 63C, pp.1-9. 10.1016/j.neuropsychologia.2014.08.002 . inserm-01067954

\section{HAL Id: inserm-01067954 https://www.hal.inserm.fr/inserm-01067954}

Submitted on 24 Sep 2014

HAL is a multi-disciplinary open access archive for the deposit and dissemination of scientific research documents, whether they are published or not. The documents may come from teaching and research institutions in France or abroad, or from public or private research centers.
L'archive ouverte pluridisciplinaire HAL, est destinée au dépôt et à la diffusion de documents scientifiques de niveau recherche, publiés ou non, émanant des établissements d'enseignement et de recherche français ou étrangers, des laboratoires publics ou privés. 
Mental simulation of future scenarios in transient global amnesia

Aurelija Juskenaite ${ }^{1234}$, Peggy Quinette ${ }^{* 1234}$, Béatrice Desgranges ${ }^{1234}$, Vincent de La Sayette $^{1235}$, Fausto Viader ${ }^{1235}$ and Francis Eustache ${ }^{1234}$

* These two authors contributed equally to this study

${ }^{1}$ U1077, INSERM, Caen, France

${ }^{2}$ UMR-S1077, Unicaen, Caen, France

${ }^{3}$ UMR-S1077, Ecole Pratique des Hautes Etudes, Caen, France

${ }^{4}$ U1077, Caen University Hospital, Caen, France

${ }^{5}$ Neurology Department, Caen University Hospital, Caen, France

Correspondence should be addressed to: Pr. Francis Eustache, Unité de recherche U1077, "Neuropsychologie et neuroanatomie fonctionnelle de la mémoire humaine", CHU, Avenue de la Côte de Nacre, 14033 Caen Cedex 09, France

Tel.: +33(0)231065197

Fax: + 33(0)231065198

E-mail: neuropsycho@chu-caen.fr 


\section{Summary}

Researchers exploring mental time travel into the future have emphasised the role played by episodic memory and its cerebral substrates. Recently, owing to controversial findings in amnesic patients, this role has become a matter of intense debate. In order to understand whether episodic memory is indeed crucial to future thinking, we assessed this ability in 11 patients during an episode of transient global amnesia (TGA), a unique and severe amnesic syndrome that primarily affects episodic memory. In the first of two experiments, TGA patients were asked to recall personal past events as well as to imagine personal future events, without any guidance regarding content. In this condition, compared with controls, they provided fewer past and fewer future events, and the latter were less closely related to their personal goals. Furthermore, TGA patients' descriptions of past and future events were scant, containing fewer descriptive elements in total and fewer internal details. In order to assess whether TGA patients might have been basing their future event narratives on their general knowledge about how these events usually unfold, in our second experiment, we asked them to imagine future events in response to short descriptions of common scenarios. In this condition, inherently eliciting less detailed descriptions, not only were all the TGA patients able to describe common events as happening in the future, but their narratives contained comparable amounts of internal detail to those of controls, despite being less detailed overall. Taken together, our results indicate that severe amnesia interferes with TGA patients' ability to envisage their personal past and future on a general level as well as in detail, but less severely affects their ability to imagine common scenarios, which are not related to their personal goals, probably owing to their preserved semantic memory, logical reasoning and ability to create vivid mental images. 
Keywords: Transient Global Amnesia; Mental Time Travel; Mental Simulation of the Future; Episodic Memory. 


\section{Introduction}

The concept of mental time travel as a freestanding ability (Suddendorf \& Corballis, 1997; Tulving, 1985) has emerged alongside the observation that amnesic patients display disturbed mental simulation of both past and future events. In the first case study to address this issue, Tulving (1985) reported that Patient KC (at that time referred to as NN), who suffered from severe amnesia following a motorcycle accident, was unable to say what he had been doing the previous day and what he would be doing the following day. Tulving (1985) therefore concluded that it is episodic memory that makes mental time travel possible, not only into the past, but also into the future. Subsequently, several other case studies featuring informal assessments have also found that patients with amnesia acquired during adulthood are impaired when it comes to evoking past or future events and situating them in time (Andelman, Hoofien, Goldberg, Aizenstein, \& Neufeld, 2010; Klein, Loftus, \& Kihlstrom, 2002; Tulving, 1985). However, the authors of these studies concluded that while the ability to recall past events and the ability to mentally simulate future events are related, the strength of this relationship is open to question (Andelman et al., 2010).

While informal assessments have mainly focused on appraising amnesic patients' ability to evoke past and future events and quantifying these events, systematic assessments of the content of such events have allowed researchers to gain insight into their qualitative characteristics. For example, a study of eight amnesic patients suffering from adult-acquired amnesia after medial temporal lobe damage showed that they produced fewer episodic details than controls, but a similar number of semantic details when asked to imagine specific past and future events within a supplied framework (Race, Keane, \& Verfaellie, 2011). On the strength of a positive correlation between past and future event scores, the authors inferred that the recall of past events and the imagination of future ones involve a common cognitive 
mechanism sustained by the medial temporal lobe, although they were unable to conclude on its precise nature.

Cognitive and neuroimaging studies of healthy individuals report that the recall of past events and the imagination of future events undergo the same developmental progression, depend on the same variables and are mediated by similar brain regions (for reviews, see, for example, Schacter et al., 2012, and Viard et al., 2012). While there seems to be a consensus on the link between these two abilities, we still do not know whether they are bound together in a relation of dependence or else simply share a common cognitive mechanism.

In support of the latter hypothesis, Hassabis and Maguire (2007) reported that when five patients with amnesia acquired after bilateral hippocampal damage were asked to imagine new scenes in response to short descriptions of common scenarios, they had difficulty producing content details of all types (i.e., spatial references, entities present, thoughts/emotions/actions, and sensory descriptions). The authors argued that the hippocampus supports the process whereby a coherent spatial context is created in which different elements of an experience can be bound, thus influencing not only the recall of past events, but also the mental simulation of fictitious events.

A contradictory conclusion was drawn by Squire et al. (2010) when they examined six patients with adult-acquired amnesia resulting from medial temporal lobe damage that included the hippocampus. No difference was found between patients and controls when it came to the unguided imagination of future events, as both groups provided comparable amounts of internal and external detail, which can be roughly equated to episodic and semantic information. The authors therefore concluded that only the recall of recent past events depends on the hippocampus, and that the recall of remote past events and the imagination of future events rely on other brain regions. However, it remains unknown whether the residual hippocampal tissue in these patients is functional to a degree sufficient to 
support imagination of new events, as it has already been reported in a patients with adultacquired amnesia (Mullally et al., 2012). Alternatively, as Maguire and Hassabis (2011) have already underlined, the discrepant results reported in this study can also be explained by the fact that the patients suffered from less severe amnesia, with intact remote memory and only modestly impaired recent autobiographical memory. In addition, this study included older patients and healthy volunteers, who are known to describe past as well as future events in a less detailed manner than young adults do (Addis, Wong, \& Schacter, 2008; Gaesser, Sacchetti, Addis, \& Schacter, 2011; Madore, Gaesser, \& Schacter, 2014; Schacter, Gaesser, \& Addis, 2013).

As in research on adult-acquired amnesia, studies of developmental amnesia do not always report convergent results. Nonetheless, difficulties imagining new events generally seem to be less visible in developmental amnesia, probably because the memory impairment is more moderate. In their study, Cooper et al. (2011) reported that 21 children with developmental amnesia displayed an unimpaired ability to imagine new familiar events when a framework was supplied for them. The authors attributed this preserved ability to the patients' semantic memory and/or a degree of functionality in their residual hippocampi. The same conclusion was reached both by Maguire et al. (2010), who showed that Patient Jon's ability to imagine new familiar events when supplied with a general framework was intact, and by Hurley et al. (2011), who reported similar results on the same task for HC, a patient suffering from developmental amnesia with bilateral hippocampal damage. However, when HC was assessed in an earlier study, using a different method that required the unguided mental simulation of future events, she produced a comparable amount of external detail to controls, but a smaller amount of internal (episodic) detail (Kwan, Carson, Addis, \& Rosenbaum, 2010). 
Overall, research reports on amnesia are difficult to compare and the symmetry between the ability to recall past events and to imagine new events is not always evidenced. Discrepancies between the results may be due to 1) differences in the patients' neuropsychological profiles, namely the severity of their anterograde amnesia, the extent of their retrograde amnesia, and the point in their lives when the memory disorders were acquired, or 2) the tasks that are used. Amnesic patients are generally asked to mentally simulate new events in three different conditions, that is to say, either in an unguided mental simulation condition, or else in response to short scenario descriptions, which in turn can involve either specific events or familiar/common events. In addition, scoring procedures tend to vary considerably, depending on the authors' hypotheses regarding the cognitive processes underlying the imagination of new events.

Studies of amnesia are, however, particularly useful for tackling questions about the concept of mental time travel and, more specifically, the role played by the ability to remember the past in the ability to imagine future events. Of particular interest here, owing to the purity, severity and recentness of the memory impairment, transient global amnesia (TGA) is a prime model of an acquired memory disorder (Bartsch et al., 2010). This neurological syndrome, of elusive aetiology, occurring suddenly and lasting up to 24 hours, is characterized by massive episodic memory impairment, but executive functions are considered to be largely spared (Quinette et al., 2003) or only slightly impaired (Jäger, Bäzner, Kliegel, Szabo, \& Hennerici, 2009). Moreover, the abrupt onset and recentness of the amnesia mean that there is no cognitive reorganization, and ensure that what looks like retrograde amnesia is not in fact long-lasting anterograde amnesia. Therefore, for the very first time, we assessed the ability of patients in the acute or peri-acute phase of TGA to project themselves into the future.

\section{Participants}


Eleven patients meeting Hodges and Warlow's (1990) clinical criteria for TGA took part in the study, seven in Experiment 1 and four in Experiment 2. All the attacks were witnessed and reported by an observer, and were characterized by clear-cut anterograde amnesia sparing other cognitive functions, and by the absence of identity loss, neurological symptoms and epileptic features, as well as the absence of recent head injury or active epilepsy. Finally, they all resolved within 24 hours. All the TGA patients, admitted to the emergency department of Caen University Hospital, underwent a neurological examination, brain CT scans and electroencephalograms. All of these were normal, except for Patient P05 in Experiment 1, whose scan revealed an occipital meningioma, apparently unrelated to the TGA episode. Patients were examined during the acute or peri-acute phase of TGA, the latter being characterized by the absence of repetitive questioning, reduced temporal disorientation, and slightly improved, but still pathological, performances on free recall tasks. The acute phase, which lasted 4-9 hours $(M=6.4, S D=2.5$ hours) for our patients, was deemed to start when the first signs of amnesia were noticed by a witness, and to end when the first memories were encoded by the patients after the onset of amnesia. All the patients and controls gave their written informed consent to take part in the research, which was approved by the local ethics committee. TGA patients gave their consent during the acute phase, and always confirmed their decision after recovery.

\section{Experiment 1: Unguided Mental Simulation of Personal Future}

\subsection{Neuropsychological assessment}

Seven TGA patients and fourteen healthy volunteers matched with the patients on age and education level took part in Experiment 1 (see Table 1 for demographic and neuropsychological data). At their point of inclusion in the study, all the patients were undergoing their first ever TGA episode, except for P07, for whom it was the second. All the patients were examined during the acute or peri-acute phase. The participants' episodic 
memory was assessed with the E/S/R task (Eustache et al., 1999). Neuropsychological assessments confirmed the presence of a massive impairment of episodic memory in all the patients during the TGA episode (see Table 1). 


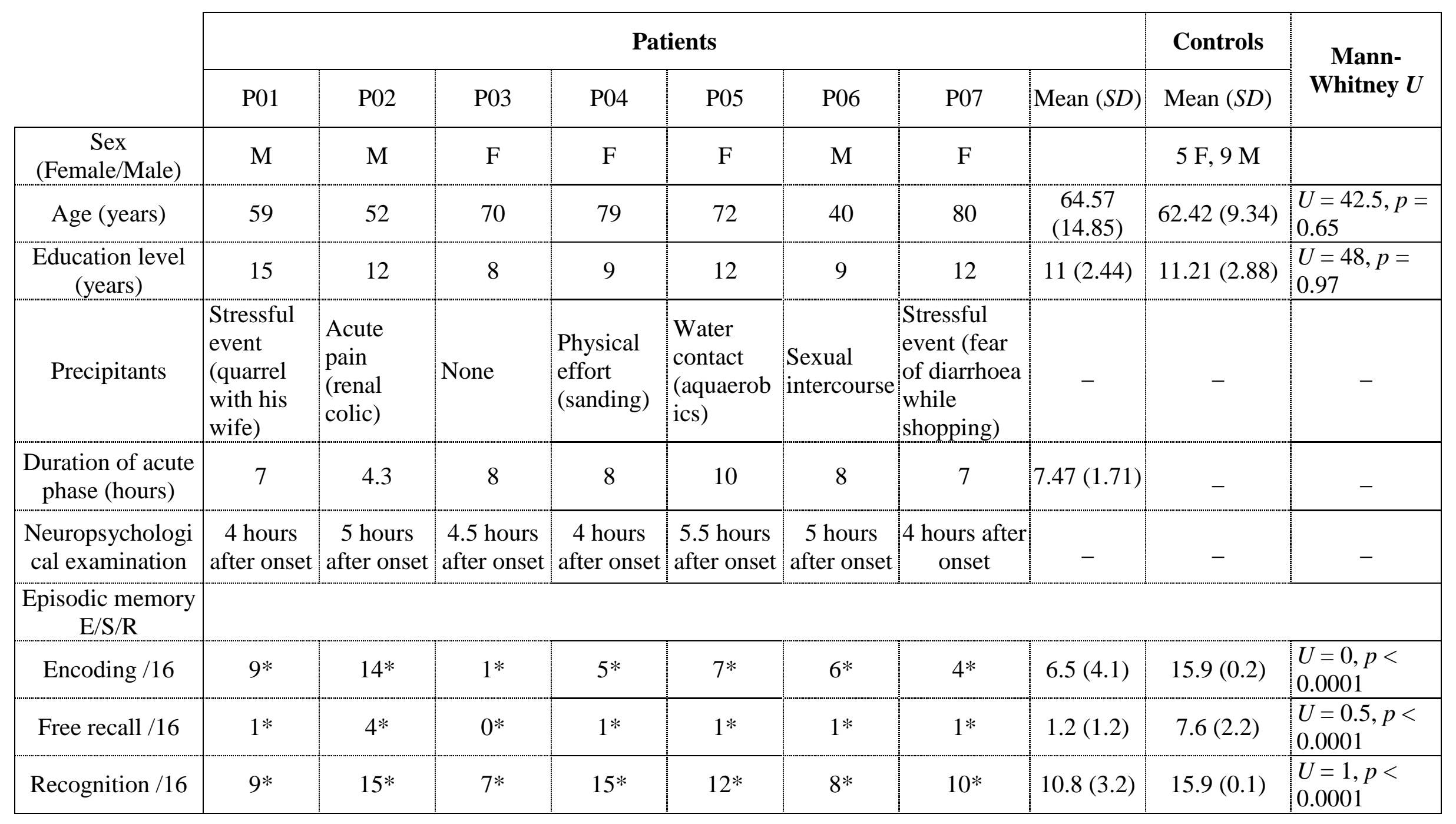

Table 1. Demographic and Neuropsychological Data of Participants in Experiment 1.

$* z<-1.65$ for patients' performances (raw scores) compared with those of controls. 


\subsection{Original task}

The task used in Experiment 1 was designed to assess the ability to project oneself into one's personal future and personal past. Participants were asked to report two different personal events for each of the following four time periods: the previous five years except for the last twelve months, the previous twelve months, the next twelve months, and the next five years except for the next twelve months. For each trial, a time period and a cue word were displayed on a computer screen, the cue being supplied to help, rather than constrain, the generation of the event, which could be unrelated to the cue. Eight emotionally neutral cue words (leg, key, bus, suitcase, storm, tea, sand, dishes) were selected from our database of 576 words (Noël et al., 2008) for their capacity to trigger specific memories, and matched for frequency, length, level of concreteness and level of imageability. The order of the four time periods, as well as of the eight cue words, was counterbalanced across participants.

Participants were told to describe out loud, and in as much detail as possible, personal events that had to be unique (i.e., not repeated in time) and take place within a single day and in a particular space and time. It was emphasized that the future events should be novel and not similar to any actual past event. If some of these future events had been scheduled, participants were encouraged to elaborate on their plans, and to pre-experience the event in as much detail as possible. Examples of appropriate and inappropriate responses were supplied. If participants strayed away from specifics, the examiner reminded them to focus on one particular event. Each trial lasted 3 minutes. If the participants' descriptions petered out before the allotted 3 minutes were up, general probing was used to elicit additional information about the episode without introducing any new idea, concept or entity.

We began by counting the number of events produced by participants in each of the four time periods, as well as for the past and future conditions overall. We then assessed the content of the participants' narratives using a modified version of the Autobiographical Memory Interview, the 
scoring procedure originally proposed by Levine and co-workers (Levine et al., 2002) that consists in counting the number of elements belonging to different content categories. Elements belonging to the main event being described were categorized as internal details, and were further subdivided into event, place, time, perceptual, and thought/emotion subcategories. Elements related to events other than the main one were classified as external details, while the semantic information category contained elements referring to general knowledge or extended states of being (e.g., "because he had been working here for 25 years"). In addition, we included a category of elements that were explicitly extracted from typical scripts (e.g., "we usually arrive on Saturday morning") and a category of optional scenarios that were only mentioned in passing in the narratives (e.g., in the sequence "I'll take a taxi ... or my daughter may drive me home ... but lets' say I'll take a taxi", the optional scenario is "or my daughter may drive me home"). Finally, for each event, we counted the total number of descriptive elements it contained and the number of probes the experimenter used.

Participants' narratives were coded by the first two authors. After all the narratives had been scored by the first author, the second author scored a random selection representing $20 \%$ of all narratives, without knowing which group they belonged to. Cronbach's $\alpha$ ranged from 0.78 to 0.912 for all content details, indicating a high agreement between scorers.

\subsection{Results}

\section{Intergroup comparisons: Number of events}

Experiment 1 revealed that when they were asked to describe past and future events without being supplied with a framework, TGA patients were frequently unable to do so, unlike controls. Analyses performed with the nonparametric Mann-Whitney $U$ test showed that, compared with controls, amnesic patients produced fewer events in the past condition $(U=15, p=.009)$ and fewer events in the future condition $(U=15, p=.009)$ as well as fewer events for each of the four time periods. Although TGA patients described comparable numbers of events for each of the four time periods, there were no correlations between them. 
When we looked at the past and future events described by participants, content analyses (see Fig. 1) showed that, despite benefiting from more numerous probes than controls (past: $U=6$, $p=.001$; future: $U=0, p<.0001$ ), TGA patients still produced fewer descriptive elements in total in both temporal conditions (past: $U=12, p=.011$; future: $U=5.5, p=.0009$ ). This included significantly fewer internal details (past: $U=13.5, p=.015$; future: $U=4, p=.0006$ ) and elements of semantic information (past: $U=1, p=.0001$; future: $U=1, p=.0001$ ), although the two groups produced comparable numbers of external details. It is worth noting that while the number of semantic details was significantly higher than the number of external details in the control group (past: $t=0, p=.0009$; future: $t=0, p=.0009$ ), the numbers were comparably small in the TGA patient group. Finally, general scripts were used neither by TGA patients nor by controls, but participants from both groups included comparably small numbers of optional scenarios in their descriptions of future events.
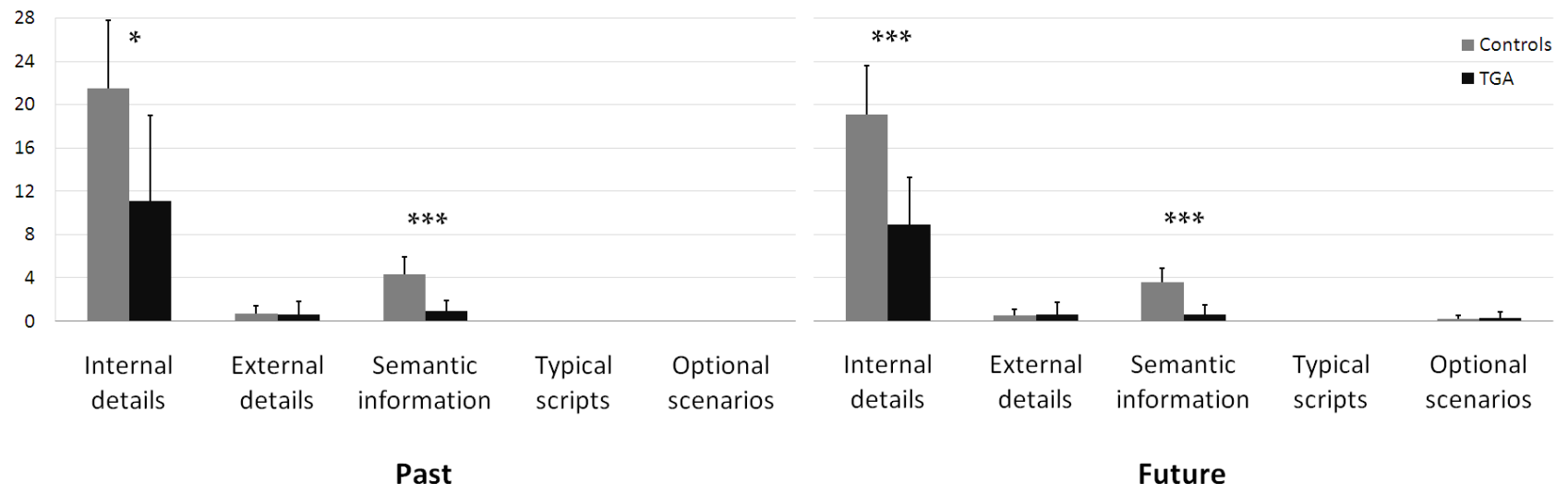

Figure 1. Mean Numbers (SD) of Internal Details, External Details, Elements of Semantic Information, Typical Scripts and Optional Scenarios Produced by Patient and Control Groups in the Past and Future Conditions.

$* p<.05, * * * p<.001 . P$ values for results of intergroup analyses.

A closer analysis of internal details revealed that, for both groups, there were more elements in the events subcategory than in any other internal detail subcategory (see Fig. 2). However, TGA patients produced significantly fewer of these elements than controls in both temporal conditions (past: $U=11.5, p=.008$; future: $U=5, p=.0009$ ). This was also the case for the thoughts/emotions 
subcategory (past: $U=13, p=.015$; future: $U=16.5, p=.032$ ) and tended to be the case for time (past: $U=17.5, p=.04$; future: $U=18, p=.05$ ). No difference was found between groups regarding the place subcategory in either temporal condition.

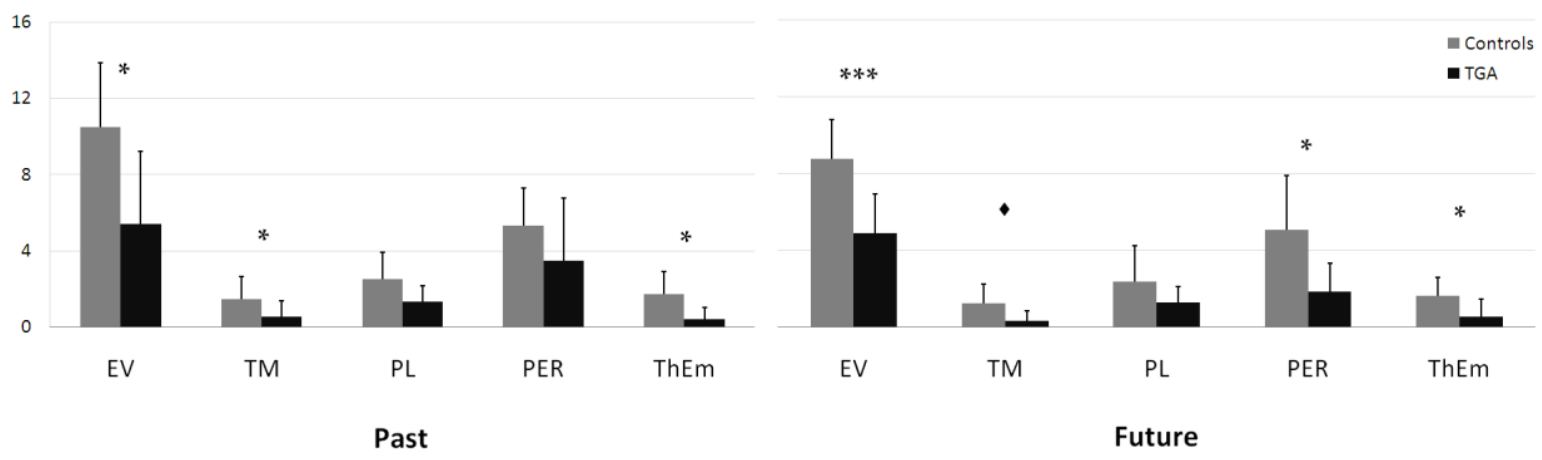

Figure 2. Mean numbers of Elements (SD) Produced for each Internal Detail Subcategory by Patient and Control Groups for Past and Future Periods.

Note. Subcategories: Events (EV), Time (TM), Place (PL), Perceptual (PER), and Thoughts/Emotions (ThEm).

$* p<.05, * * * p<.001,{ }^{*} p=.05 . P$ values for results of intergroup analyses.

\section{Content comparisons between past and future}

Intragroup analyses of the event descriptions carried out simply to compare the descriptions of past and future events showed that controls produced fewer descriptive elements in total $(t=17$, $p=.046)$ and tended to produce fewer internal details $(t=21.5, p=.051)$, with significantly fewer elements in the event subcategory $(t=21, p=.047)$, for the future condition than for the past one. Positive correlations were found between the past and future conditions in the control group regarding the number of descriptive elements in total $(r=.69, p=.005)$ and the number of internal details $(r=.78, p=.0009)$. By contrast, while no difference was found in the TGA patient group between past and future periods for any detail category or internal detail subcategory, no correlations between the two temporal conditions were evidenced. The number of perceptual details did not differ between the past and future conditions in either group. Compared with controls, TGA 
patients produced fewer of these details for future events $(U=13, p=.015)$, but not for past events $(U=27, p=.23)$.

\subsection{Discussion of Experiment 1}

In Experiment 1, where participants were asked to recall events they had experienced in the past and imagine events they might experience in the future, without any guidance regarding content, TGA patients produced fewer events for both temporal conditions than controls, and frequently failed to give any description at all. While our results are in line with previous findings reporting poorer recall of autobiographical events by TGA patients compared with controls, especially for the recent past (Guillery-Girard et al., 2004; Bartsch, Döhring, Rohr, Jansen, \& Deuschl, 2011), they also provide the first evidence of the difficulties that these patients encounter when they attempt to imagine the future.

The small number of past and future events produced by the TGA patients indicates that they struggled to establish a general framework for these events. Research suggests that during the process of generating specific past and future events, only a small proportion of them are directly accessed (Anderson et al., 2012; Anderson \& Dewhurst, 2009; D’Argembeau \& Mathy, 2011; Haque \& Conway, 2001), and semantic personal knowledge is generally retrieved first, with the episodic details arriving later (D’Argembeau \& Mathy, 2011). This general personal knowledge, including information about one's personal future goals, anticipated lifetime periods and general future events, probably provides a context for specific past and future autobiographical events, linking and organizing them according to broader themes and sequences (D'Argembeau \& Demblon, 2012; Demblon \& D'Argembeau, 2014). Retrograde amnesia in TGA would appear to render information about patients' past lives, or at least parts of them, temporarily unavailable (Eustache et al., 1999; Guillery-Girard et al., 2004) and interferes with the construction of a general framework not only for past events, but also for future ones, as personal knowledge regarding the future was also encoded in the past. 
Qualitative analysis showed that $78 \%$ of future events produced by controls were events that they either already had planned or else wished to happen sometime in the future, while the remaining $22 \%$ were products of pure imagination, often elaborating on specific circumstances of their usual activities. Not only did TGA patients produce fewer events than controls, but only 53\% of those events consisted of personal plans or wishes regarding the future - a significantly lower proportion than that of the control group $\left(x^{2}(1)=12.9, p<.001\right)$. Taken together, our results suggest that TGA patients have difficulty accessing their personal knowledge about the future, but may compensate for this lack by elaborating on their daily activities and imagining them as happening later in time. It therefore seems that accessing personal knowledge is the only option for constructing a general framework for past events, though not for future ones, and it is the one that is primarily affected by episodic memory disorders. This imbalance may explain the absence of a correlation between the numbers of past and future events provided by TGA patients, indicating that the relationship between the construction of a general framework for past events and the construction of a general framework for future ones is somewhat complex.

Analysis of the content of described events showed that despite receiving more probes than controls, TGA patients produced fewer internal details, semantic details and descriptive elements in total, in both the past and future conditions. TGA patients' difficulty keeping track of what they had already described, owing to their rapid forgetting, probably resulted in spontaneously shorter narratives containing fewer internal details, and therefore in more frequent probing. As far as semantic information is concerned, controls seem to have used it to render their descriptions of events more meaningful, by putting them into a context of reality, and make them easier for the experimenter to understand (e.g., "because he had been working here for 25 years" or "we will be grandparents by that time"). The fact that TGA patients produced fewer semantic details than controls may be directly related to the shortness of their narratives, resulting in fewer elements needing to be explained. 
While the content of TGA patients' narratives has never previously been examined in detail, studies have already shown that their narratives of past events are less detailed overall than those of controls (Bartsch, Döhring, Rohr, Jansen, \& Deuschl, 2011; Evans, Wilson, Wraight, \& Hodges, 1993; Guillery-Girard et al., 2004; Hodges \& Ward, 1989). Our results in TGA indicate that severe amnesia limits patients' detailed elaboration of past and future events in a similar manner. When past and future conditions were compared, no difference was found in the number of elements belonging to any detail category for the TGA group, whereas controls produced fewer descriptive elements in total, and tended to produce fewer internal details for future events than for past ones. This pattern of performance among healthy individuals has been repeatedly reported in the literature (Addis, Musicaro, Pan, \& Schacter, 2010; Addis et al., 2008; D’Argembeau, Ortoleva, Jumentier, \& Van der Linden, 2010; D’Argembeau \& Van der Linden, 2004; Hill \& Emery, 2013), and can be explained by higher cognitive demands related to the elaborative process during the imagination of future events. The same phenomenon may also account for why TGA patients produced fewer perceptual details than controls for the future, but not for the past.

This first experiment highlighted the difficulty that TGA patients have providing detailed descriptions of past as well as future events. It did not, however, corroborate the results reported in Squire and co-workers' study (2010) for older patients suffering from permanent amnesia, despite using the same task. This may be due to differences in the severity of amnesia between the patients in these two studies. TGA patients exhibit severe amnesia with a sudden onset that precludes the cerebral reorganization and cognitive compensatory processes that may have taken place in the patients assessed in Squire and coworkers' study, as they had been living with amnesia for many years. Furthermore, the latter study failed to find any significant difference between the groups regarding the number of internal details for the remote past and future periods, with a trend toward significance for the recent past period, indicating only moderate memory impairment in these patients. 
While their detailed recollection of personal past events and detailed imagination of personal future events was impaired, the TGA patients in our experiment still produced constrained descriptions of events, probably using their logical reasoning and semantic knowledge (Jäger et al., 2009) about how certain events usually unfold to help them maintain a coherent storyline. In order to test this hypothesis, we asked another group of TGA patients and controls to imagine familiar, everyday scenarios taking place in the future, and to describe them in detail. In addition, in order to examine whether TGA patients' poor performances could be due to difficulty generating vivid mental images per se, we assessed participants' imageability using the Vividness of Visual Imagery Questionnaire (Marks, 1973).

\section{Experiment 2: Mental Simulation of Common Future Scenarios}

\subsection{Neuropsychological assessment}

Four different TGA patients and seven different healthy volunteers, matched with the patients on age and education level, took part in Experiment 2 (see Table 2 for descriptive and neuropsychological data). At their point of inclusion in the study, all the patients were undergoing their first ever TGA episode. All the patients were examined during the acute or peri-acute phase. The neuropsychological assessment of the participants was the same as in Experiment 1.

\begin{tabular}{|c|c|c|c|c|c|}
\hline & \multicolumn{4}{|c|}{ Patients } & \multirow{2}{*}{$\begin{array}{c}\text { Controls } \\
\text { Mean }(S D)\end{array}$} \\
\hline & P01 & $\mathrm{P} 02$ & P03 & P04 & \\
\hline Sex (Female/Male) & $\mathrm{F}$ & $\mathrm{F}$ & $\mathrm{F}$ & M & $5 \mathrm{~F}, 2 \mathrm{M}$ \\
\hline Age (years) & 62 & 54 & 76 & 59 & $65.36(4.26)$ \\
\hline Education level (years) & 9 & 17 & 15 & 7 & $10.43(2.23)$ \\
\hline Precipitants & $\begin{array}{l}\text { Physical } \\
\text { effort in the } \\
\text { garden }\end{array}$ & $\begin{array}{c}\text { Contact with } \\
\text { water } \\
\text { (swimming } \\
\text { pool) }\end{array}$ & $\begin{array}{c}\text { Stressful } \\
\text { event (bad } \\
\text { news from } \\
\text { her } \\
\text { daughter) }\end{array}$ & None & --- \\
\hline $\begin{array}{c}\text { Duration of acute TGA } \\
\text { phase (hours) }\end{array}$ & 8.25 & 4 & 9 & 4.5 & --- \\
\hline $\begin{array}{l}\text { Neuropsychological } \\
\text { examination }\end{array}$ & $\begin{array}{c}3 \text { hours after } \\
\text { onset }\end{array}$ & $\begin{array}{c}2 \text { hours after } \\
\text { onset }\end{array}$ & $\begin{array}{l}5 \text { hours } \\
\text { after onset }\end{array}$ & $\begin{array}{l}5 \text { hours } \\
\text { after onset }\end{array}$ & --- \\
\hline
\end{tabular}




\begin{tabular}{|c|c|c|c|c|c|}
\hline \multicolumn{6}{|l|}{$\begin{array}{c}\text { Episodic memory } \\
\text { E/S/R }\end{array}$} \\
\hline Encoding /16 & $11 *$ & $9 *$ & $13 *$ & $16^{*}$ & $16(0)$ \\
\hline Free recall /16 & $1 *$ & $2 *$ & $2 *$ & $5^{*}$ & $10.25(0.96)$ \\
\hline Recognition /16 & $5^{*}$ & 8* & $7 *$ & $12^{*}$ & $16(0)$ \\
\hline
\end{tabular}

Table 2. Demographic and Neuropsychological Data of Participants Included in Experiment 2.

$* z<-1.65$ for patients' performances (raw scores) compared with those of controls.

\subsection{Original task}

In our second experiment, participants were invited to imagine four events in the way they might experience them at some point within the next 5 years. In order to provide a general framework for these events, we supplied participants with cue-sentences representing common scenarios: "You will be invited to a friend's birthday", "You will change your phone number", "You will be invited to celebrate a retirement", "You will go to visit a friend in Paris". Participants were told that after reading each cue-sentence, they would have to imagine the event as happening in a particular time and place in the future, within the space of a single day, sometime within the next 5 years, and describe it out loud in as much detail as possible. It was emphasized that the imagined events had to be unique and novel ones that could not be repeated, and which did not resemble any events that had taken place in the past. Each trial lasted 3 minutes, and if the participants' descriptions petered out before this time was up, general probing was used to elicit additional details about the episode, taking care not to introduce any new idea, concept or entity. The instructions and scoring procedure used in this experiment were the same as those used in Experiment 1 .

In addition, participants answered the Vividness of Visual Imagery Questionnaire (VVIQ) while keeping their eyes open, in order to assess their ability to create vivid images in their minds.

\subsection{Results}

Intergroup comparisons: Content analysis 
The results of Experiment 2 indicated that when a general framework was supplied, all the participants were able to describe future events, with no difference in their number between the two groups. As in Experiment 1, we compared the groups on the content of the events, using the nonparametric Mann-Whitney $U$ test. Statistical analyses showed that TGA patients and controls benefited from comparable numbers of probes, but the former produced fewer descriptive elements in total $(U=0, p=.006)$. The difference between the groups in the number of internal details failed to reach statistical significance $(U=4, p=.07)$, and no difference was found regarding the number of semantic details, although the number of external details was significantly lower in the patient group than in the control group $(U=0, p=.006)$. It is interesting to note that in the control group, the internal detail category contained the highest number of elements, but in contrast to Experiment 1, no difference was found between the numbers of external and semantic details. In the patient group, although internal details tended to outnumber other ones, no significant difference was found between any of the detail categories. Finally, TGA patients and controls produced comparably low numbers of typical scripts and optional scenarios (see Fig. 3).

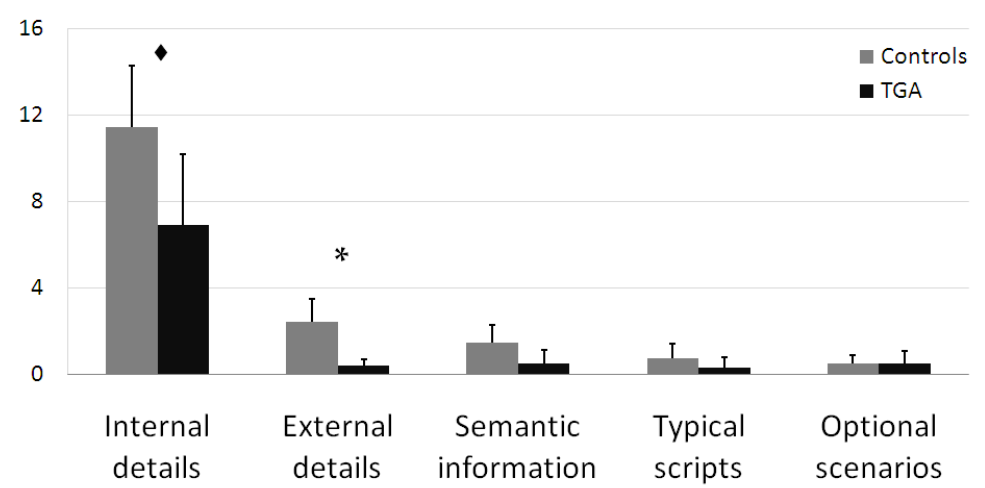

Figure 3. Mean Numbers (SD) of Internal Details, External Details, Elements of Semantic Information, Typical Scripts and Optional Scenarios, Averaged Across the Four Future Events for the TGA and Control Groups.

$* p<.05, p=.07 . P$ values for results of intergroup analyses. 
Analyses of internal detail subcategories showed that TGA patients produced fewer elements than controls for the time $(U=3.5, p=.042)$, place $(U=.5, p=.006)$ and perceptual $(U=3, p=.042)$ subcategories (see Fig. 4).

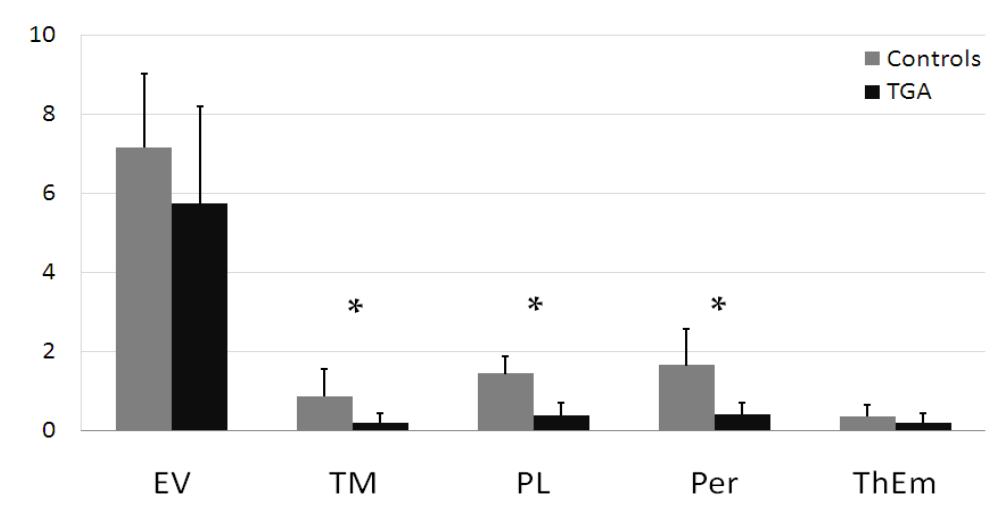

Figure 4. Mean Numbers of Elements (SD) Produced for each Internal Detail Subcategory by Patient and Control Groups.

Note. Subcategories: Events (EV), Time (TM), Place (PL), Perceptual (PER), and Thoughts/Emotions (ThEm).

$* p<.05 . P$ value for results of intergroup analyses.

Finally, the TGA patients' performances on the VVIQ questionnaire did not differ from those of controls.

\subsection{Discussion of Experiment 2}

All the TGA patients were able to describe events for which a general framework had been supplied. This result indicates that severe amnesia in TGA does not prevent patients from envisaging the unfolding of commonplace events, which is consistent with clinical observations of intact logical reasoning during the acute phase of TGA, even anticipating the future (e.g., taking appropriate documents and other belongings when told that they are going to hospital). There seems to be a consensus about the preservation of the ability to provide more or less detailed descriptions of events for which a framework is supplied in studies of patients with either adult-acquired 
(Hassabis et al., 2007; Race et al., 2011) or developmental amnesia (Cooper et al., 2011; Hurley et al., 2011; Maguire et al., 2010).

The content of the events provided in this second experiment indicates that controls imagined these familiar, everyday scenarios of little personal significance with fewer details than those they had imagined freely in Experiment 1. While controls produced more internal details than any other category in both experiments, there were only about half as many for common events as there had been for personal events. This decrease in internal details was accompanied by a decrease in semantic details, which were also halved in number, no longer outnumbering external details. This is not surprising, given that semantic information is usually used to explain or contextualize the main event.

Similarly, analyses of the TGA patients' descriptions revealed a smaller ratio of internal details for common events than for personal ones, as they no longer significantly outnumbered details belonging to other content categories. Finally, in contrast to Experiment 1, participants in Experiment 2 used information that had been explicitly extracted from typical scripts ("we usually arrive on Saturday morning"), in addition to the fleeting mention of optional scenarios ("or my daughter might drive me home").

While no studies, to our knowledge, have directly compared imagination for personal future events without any content guidance with imagination for cued future common events, it has already been suggested that amnesic patients tend to generate a greater number of details when imagining frequent events, compared with less frequent ones (Race et al., 2011). Our study not only indicates that this pattern of results may apply to controls, as well as to amnesic patients, but also suggests that the cognitive process underlying imagination for cued common events is not severely affected by amnesia. For although TGA patients produced fewer descriptive elements in total than controls, the difference between the two groups regarding the number of internal details failed to reach statistical significance, even though the TGA patients produced fewer elements than controls 
for three of the five internal detail subcategories. The two groups also produced comparable numbers of semantic details.

In contrast to our results, Race et al. (2010) found that patients with permanent amnesia acquired during adulthood produced fewer internal details and fewer details in all the internal detail subcategories than controls. This can be explained by the fact that their study was designed to elicit specific, rather than commonplace events (albeit varying in frequency), in order to minimize descriptions of repeated events or frequently retrieved event information (Race et al., 2011). When such event information is accessible, TGA patients seem to be able to use it to describe future events on a relatively general level just as successfully as controls.

When they are asked to describe cued events involving familiar, everyday scenarios of little personal significance, TGA patients are quite capable of imagining them taking place in the future, probably relying on their cognitive schemas and semantic memory in general, which are known to be preserved in this pathology (Jäger et al., 2009) - as is their ability to create vivid mental images, witness their performances on the VVIQ questionnaire.

\section{General Discussion}

The aim of the present study was to enhance current understanding of the cognitive processes involved in the mental simulation of future events and their disturbance resulting from severe episodic memory deficit. To this end, we asked patients undergoing a TGA episode, characterized by the rapid onset of severe anterograde and retrograde amnesia, to recall events they had experienced in the past and to imagine events they might experience in the future, without any content guidance. In these conditions, TGA patients produced fewer events than controls in both past and future conditions.

Various informal assessments of single patients with adult-acquired permanent amnesia have revealed that these patients have difficulty projecting themselves into their personal future (Andelman et al., 2010; Klein et al., 2002; Tulving, 1985). This difficulty was also briefly mentioned in a systematic group study (Squire et al., 2010), which unfortunately did not indicate 
whether the amnesic patients provided significantly fewer events than controls. However, the small number of studies in amnesia comparing imagination for past and future events without any content guidance precludes extensive analysis of the mental processes involved in these tasks. Our study was thus an attempt to gain a better understanding of this issue.

Data in the literature suggest that when asked to provide specific personal events, healthy volunteers mostly activate general autobiographical knowledge first, followed by the production of episodic details, and this is true for both past and future events (D'Argembeau et al., 2011; Haque \& Conway, 2001). It has been suggested that memory for personal events relies on these general knowledge structures, which frame episodic details within the individual's autobiography, organized into coherent themes and sequences (D'Argembeau \& Demblon, 2012; Demblon \& D’Argembeau, 2014).

Autobiographical knowledge must be accessed in order to construct a framework for a personal past event. In the case of future events, our qualitative analysis showed that their general framework can be constructed either by accessing personal knowledge about events that are already planned or desired, or alternatively by imagining a more or less routine activity taking place in the future. In all likelihood, the latter strategy relies more on semantic knowledge and logical reasoning than on autobiographical knowledge, which is probably why it was more frequently used by TGA patients (i.e., patients suffering from retrograde amnesia) than by controls. We suggest that differences in the mental strategies available for constructing frameworks for recollecting past events or imagining future ones - strategies that seem to be impacted differently by episodic memory disorders - account for the absence of correlations between the numbers of past and future events produced by TGA patients.

Even when TGA patients succeeded in retrieving a general framework for a past or future event, they were less effective in elaborating its details than controls, providing fewer descriptive elements in total, as well as fewer internal and semantic details. They produced shorter and less detailed narratives in both temporal conditions, suggesting that the impact of severe amnesia on 
episodic simulation is the same for both past and future events. This pattern of results, already reported for permanent amnesia (Kwan et al., 2010; Race et al., 2011), suggests that episodic memory impairment goes hand in hand with the disturbance of a mental process that underlies the detailed simulation of both past and future events.

Three major hypotheses regarding this process have been advanced and still remain a matter of debate in the literature. The constructive episodic simulation hypothesis (Schacter \& Addis, 2007) maintains that the imagination of future events depends on the ability to access, extract and combine information from memories of past events. Similarly, it has been argued that memories of past events are used in different mental activities requiring self-projection to alternative perspectives to the present one, and this obviously applies to the imagination of future events as well (Buckner \& Carroll, 2007). Finally, the scene construction hypothesis suggests that the ability to mentally generate and maintain a complex and coherent scene is the key process underlying both the recall of past events and the imagination of future ones (Hassabis, Kumaran, Vann, \& Maguire, 2007).

However, the demonstration of comparable disturbances of past and future events is not enough to conclude on the precise nature of the cognitive mechanism whose failure leads to this pattern of results. While the latter points to a link between these two cognitive abilities, it does not allow us to determine whether one cognitive ability depends on the other, or whether a more basic mental process is engaged in both these tasks. Therefore we cannot rule out any of these hypotheses regarding the episodic simulation of events.

While our results do not contradict Hassabis and Maguire's (2009) view that time cannot be regarded as an independent process with a distinct neural signature when it comes to episodic mental simulation, they do afford us a better understanding of the construction mechanisms that generally precede this phase. Our study suggests that some of the cognitive strategies that allow for the creation of a general framework are common to personal past and personal future events, while others are specific to future events. This view is consistent with neuroimaging data showing that the 
construction phase engages a number of brain regions, some of which are exclusively recruited for past or for future events, while the elaboration phase of both types of events seems to be characterized by considerable regional overlap (Addis, 2007).

Our study showed that severe episodic memory impairment affects both these phases, but does not completely annihilate them. A general framework for personal future events can still be constructed on the basis of routine activities that can be imagined as happening in the future even when autobiographical knowledge is virtually inaccessible, and further elaboration of future events can be partially based on cognitive schemas and general semantic memory. When they were asked to imagine cued common events, our TGA patients described them in a manner that was roughly comparable to that of controls, probably making use of their intact semantic memory (Jäger et al., 2009) and their intact ability to mentally create vivid mental images.

Mental projection towards the past or towards the future, therefore seems to be a complex activity. Among other cognitive abilities, it appears to draw on semantic knowledge of general scripts and various routines to guide the elaboration of past and future events and fill in their essential details. However, it remains unclear whether a critical mass of descriptive detail amounts to proof of the episodic nature of mental simulation. Clearly identified and consensual criteria allowing us to distinguish between episodic and semantic types of mental simulation are still lacking, and represent a challenge for researchers, who will probably have to rethink the very nature and processes of episodic and semantic memory. 


\section{Funding}

This work was carried out as part of clinical research hospital programme undertaken by Caen University Hospital. The PhD funding of Aurelija Juskenaite was supported by Lower Normandy Regional Council, the Vicq d'Azyr association and INSERM.

\section{Acknowledgments}

We would like to thank Vanessa Matuszewski, Pascale Piolino, Alice Pélerin and Mathieu Hainselin for their contributions to this study, as well as the Accident and Emergency Department, Neurology Units and Clinical Research Department of Caen University Hospital for their precious collaboration. 


\section{References}

Addis, D. R., Musicaro, R., Pan, L., \& Schacter, D. L. (2010). Episodic simulation of past and future events in older adults: Evidence from an experimental recombination task. Psychology and Aging, 25(2), $369-376$.

Addis, D. R., Pan, L., Vu, M.-A., Laiser, N., \& Schacter, D. L. (2009). Constructive episodic simulation of the future and the past: Distinct subsystems of a core brain network mediate imagining and remembering. Neuropsychologia, 47(11), 2222-2238.

Addis, D. R., Wong, A. T., \& Schacter, D. L. (2008). Age-related changes in the episodic simulation of future events. Psychological Science, 19(1), 33-41.

Andelman, F., Hoofien, D., Goldberg, I., Aizenstein, O., \& Neufeld, M. Y. (2010). Bilateral hippocampal lesion and a selective impairment of the ability for mental time travel. Neurocase, 16(5), 426-435.

Anderson, R. J., \& Dewhurst, S. A. (2009). Remembering the past and imagining the future: differences in event specificity of spontaneously generated thought. Memory, 17(4), 367-373.

Anderson, R. J., Dewhurst, S. A., \& Nash, R. A. (2012). Shared cognitive processes underlying past and future thinking: the impact of imagery and concurrent task demands on event specificity. Journal of Experimental Psychology, 38(2), 356-365.

Bartsch, T., Döhring, J., Rohr, A., Jansen, O., \& Deuschl, G. (2011). CA1 neurons in the human hippocampus are critical for autobiographical memory, mental time travel, and autonoetic consciousness. Proceedings of the National Academy of Sciences of the United States of America, 108(42), 17562-17567.

Bartsch, T., Schönfeld, R., Müller, F. J., Alfke, K., Leplow, B., Aldenhoff, J., ... Koch, J. M. (2010). Focal lesions of human hippocampal CA1 neurons in transient global amnesia impair place memory. Science, 328(5984), 1412-1415.

Buckner, R. L., \& Carroll, D. C. (2007). Self-projection and the brain. Trends in Cognitive Sciences, 11(2), $49-57$. 
Cooper, J. M., Vargha-Khadem, F., Gadian, D. G., \& Maguire, E. A. (2011). The effect of hippocampal damage in children on recalling the past and imagining new experiences. Neuropsychologia, 49(7), 1843-1850.

D’Argembeau, A., \& Demblon, J. (2012). On the representational systems underlying prospection: evidence from the event-cueing paradigm. Cognition, 125(2), 160-167.

D'Argembeau, A., \& Mathy, A. (2011). Tracking the construction of episodic future thoughts. Journal of Experimental Psychology. 140(2), 258-271.

D’Argembeau, A., Ortoleva, C., Jumentier, S., \& Van der Linden, M. (2010). Component processes underlying future thinking. Memory \& Cognition, 38(6), 809-819.

D'Argembeau, A., \& Van der Linden, M. (2004). Phenomenal characteristics associated with projecting oneself back into the past and forward into the future: influence of valence and temporal distance. Consciousness and Cognition, 13(4), 844-858.

Demblon, J., \& D'Argembeau, A. (2014). The organization of prospective thinking: evidence of event clusters in freely generated future thoughts. Consciousness and Cognition, 24, 75-83.

Eustache, F., Desgranges, B., Laville, P., Guillery, B., Lalevee, C., Schaeffer, S., ... Viader, F. (1999). Episodic memory in transient global amnesia: Encoding, storage, or retrieval deficit? Journal of Neurology, Neurosurgery, and Psychiatry, 66(2), 148-154.

Evans, J., Wilson, B., Wraight, E. P., \& Hodges, J. R. (1993). Neuropsychological and SPECT scan findings during and after transient global amnesia: Evidence for the differential impairment of remote episodic memory. Journal of Neurology, Neurosurgery, and Psychiatry, 56(11), $1227-1230$.

Gaesser, B., Sacchetti, D. C., Addis, D. R., \& Schacter, D. L. (2011). Characterizing age-related changes in remembering the past and imagining the future. Psychology and Aging, 26(1), $80-84$.

Guillery-Girard, B., Desgranges, B., Urban, C., Piolino, P., de La Sayette, V., \& Eustache, F. (2004). The dynamic time course of memory recovery in transient global amnesia. Journal of Neurology, Neurosurgery, and Psychiatry, 75(11), 1532-1540. 
Haque, S., \& Conway, M. A. (2001). Sampling the process of autobiographical memory construction. European Journal of Cognitive Psychology, 13(4), 529-547.

Hassabis, D., Kumaran, D., Vann, S. D., \& Maguire, E. A. (2007). Patients with hippocampal amnesia cannot imagine new experiences. Proceedings of the National Academy of Sciences of the United States of America, 104(5), 1726-1731.

Hassabis, D., \& Maguire, E. A. (2009). The construction system of the brain. Philosophical Transactions of the Royal Society, 364(1521), 1263-1271.

Hill, P. F., \& Emery, L. J. (2013). Episodic future thought: contributions from working memory. Consciousness and Cognition, 22(3), 677-683.

Hodges, J. R., \& Ward, C. D. (1989). Observations during transient global amnesia. A behavioural and neuropsychological study of five cases. Brain: A Journal of Neurology, 112(Pt 3), 595620.

Hodges, J. R., \& Warlow, C. P. (1990). Syndromes of transient amnesia: Towards a classification. A study of 153 cases. Journal of Neurology, Neurosurgery, and Psychiatry, 53(10), 834843.

Hurley, N. C., Maguire, E. A., \& Vargha-Khadem, F. (2011). Patient HC with developmental amnesia can construct future scenarios. Neuropsychologia, 49(13), 3620-3628.

Jäger, T., Bäzner, H., Kliegel, M., Szabo, K., \& Hennerici, M. G. (2009). The transience and nature of cognitive impairments in transient global amnesia: A meta-analysis. Journal of Clinical and Experimental Neuropsychology, 31(1), 8-19.

Klein, S. B., Loftus, J., \& Kihlstrom, J. F. (2002). Memory and temporal experience: The effects of episodic memory loss on an amnesic patient's ability to remember the past and imagine the future. Social Cognition, 20(5), 353-379.

Kwan, D., Carson, N., Addis, D. R., \& Rosenbaum, R. S. (2010). Deficits in past remembering extend to future imagining in a case of developmental amnesia. Neuropsychologia, 48(11), $3179-3186$. 
Levine, B., Svoboda, E., Hay, J. F., Winocur, G., \& Moscovitch, M. (2002). Aging and autobiographical memory: Dissociating episodic from semantic retrieval. Psychology and Aging, 17(4), 677-689.

Madore, K. P., Gaesser, B., \& Schacter, D. L. (2014). Constructive episodic simulation: dissociable effects of a specificity induction on remembering, imagining, and describing in young and older adults. Journal of Experimental Psychology, 40(3), 609-622.

Maguire, E. A., \& Hassabis, D. (2011). Role of the hippocampus in imagination and future thinking. Proceedings of the National Academy of Sciences, 108, E39-E39.

Maguire, E. A., Vargha-Khadem, F., \& Hassabis, D. (2010). Imagining fictitious and future experiences: Evidence from developmental amnesia. Neuropsychologia, 48(11), 3187-3192.

Marks, D. F. (1973). Visual imagery differences in the recall of pictures. British Journal of Psychology, 64(1), 17-24.

Mullally, S. L., Hassabis, D., \& Maguire, E. A. (2012). Scene construction in amnesia: an FMRI study. The Journal of Neuroscience 32(16), 5646-5653.

Noël, A., Quinette, P., Guillery-Girard, B., Dayan, J., Piolino, P., Marquis, S., ... Eustache, F. (2008). Psychopathological factors, memory disorders and transient global amnesia. British Journal of Psychiatry, 193(2), 145-151.

Quinette, P., Guillery, B., Desgranges, B., de La Sayette, V., Viader, F., \& Eustache, F. (2003). Working memory and executive functions in transient global amnesia. Brain, 126(Pt 9), 1917-1934.

Race, E., Keane, M. M., \& Verfaellie, M. (2011). Medial temporal lobe damage causes deficits in episodic memory and episodic future thinking not attributable to deficits in narrative construction. Journal of Neuroscience, 31, 10262-10269.

Schacter, D. L., \& Addis, D. R. (2007). The cognitive neuroscience of constructive memory: remembering the past and imagining the future. Philosophical Transactions of the Royal Society of London, 362(1481), 773-786. 
Schacter, D. L., Addis, D. R., Hassabis, D., Martin, V. C., Spreng, R. N., \& Szpunar, K. K. (2012). The future of memory: Remembering, imagining, and the brain. Neuron, 76(4), 677-694.

Schacter, D. L., Gaesser, B., \& Addis, D. R. (2013). Remembering the past and imagining the future in the elderly. Gerontology, 59(2), 143-151.

Squire, L. R., van der Horst, A. S., McDuff, S. G. R., Frascino, J. C., Hopkins, R. O., \& Mauldin, K. N. (2010). Role of the hippocampus in remembering the past and imagining the future. Proceedings of the National Academy of Sciences of the United States of America, 107(44), 19044-19048.

Suddendorf, T., \& Corballis, M. C. (1997). Mental time travel and the evolution of the human mind. Genetic, Social, and General Psychology Monographs, 123(2), 133-167.

Tulving, E. (1985). Memory and consciousness. Canadian Psychology/Psychologie Canadienne, 26(1), 1-12.

Van Mulukom, V., Schacter, D. L., Corballis, M. C., \& Addis, D. R. (2013). Re-imagining the future: Repetition decreases hippocampal involvement in future simulation. PLOS ONE, 8(7), e69596.

Viard, A., Desgranges, B., Eustache, F., \& Piolino, P. (2012). Factors affecting medial temporal lobe engagement for past and future episodic events: An ALE meta-analysis of neuroimaging studies. Brain and Cognition, 80(1), 111-125. 